\title{
WHOLE-EXOME ANALYSIS IN PARKINSON'S DISEASE REVEALS A HIGH BURDEN OF ULTRA RARE VARIANTS IN EARLY ONSET CASES
}

Bernabe I. Bustos ${ }^{1}$, Dimitri Krainc ${ }^{1}$ and Steven J. Lubbe ${ }^{*}$, for The International Parkinson's Disease Genomics Consortium (IPDGC) ${ }^{\ddagger}$.

${ }^{1}$ Ken and Ruth Davee Department of Neurology and Simpson Querrey Center for Neurogenetics, Northwestern University Feinberg School of Medicine, Chicago, Illinois, USA.

${ }^{\ddagger}$ Full list of IPDGC members in pdgenetics.org/partners.

*Corresponding author: Steven J. Lubbe, PhD (steven.1ubbe@northwestern.edu)

Word count:

Abstract $=208$

Main text $=4,362(3,285$ excluding Methods $)$ 


\begin{abstract}
Parkinson's disease (PD) is a complex neurodegenerative disorder with a strong genetic component. We performed a "hypothesis-free" exome-wide burden-based analysis of different variant frequencies, predicted functional impact and age of onset classes, in order to expand the understanding of rare variants in PD. Analyzing whole-exome data from a total of 1,425 PD cases and 596 controls, we found a significantly increased burden of ultra-rare (URV= private variants absent from gnomAD) protein altering variants (PAV) in early-onset PD cases (EOPD, $<40$ years old; $\mathrm{P}=3.95 \times 10^{-26}$, beta $\left.=0.16, \mathrm{SE}=0.02\right)$, compared to LOPD cases ( $>60$ years old, late-onset), where more common PAVs (allele frequencies $<0.001$ ) showed the highest significance and effect $(\mathrm{P}=0.026$, beta $=0.15, \mathrm{SE}=0.07)$. Gene-set burden analysis of URVs in EOPD highlighted significant disease- and tissue-relevant genes, pathways and protein-protein interaction networks that were different to that observed in non-EOPD cases. Heritability estimates revealed that URVs account for $15.9 \%$ of the genetic component in EOPD individuals. Our results suggest that URVs play a significant role in EOPD and that distinct etiological bases may exist for EOPD and sporadic PD. By providing new insights into the genetic architecture of PD, our study may inform approaches aimed at novel gene discovery and provide new directions for genetic risk assessment based on disease age of onset.
\end{abstract}




\section{INTRODUCTION}

Parkinson's disease (PD) is a complex neurodegenerative disorder characterized by the loss of dopaminergic neurons in the substantia nigra (SN), leading to motor dysfunction and a progressive neurodegenerative syndrome. Several common and rare disease-associated genes and loci have been discovered in families and large case-control studies; however, they currently explain only about $26-36 \%$ of the disease heritability ${ }^{1}$, therefore additional novel variants and genes associated with PD are yet to be found.

Despite the recent explosion of available genetic data for PD, the vast majority of cases still have an unknown genetic basis ${ }^{2}$. The use of next generation sequencing (NGS) in PD has enabled largescale whole-exome sequencing (WES) studies in both families and case-controls unrelated populations $^{3}$. Several studies have leveraged the use of WES to analyze candidate genes or known biological pathways and their load of rare variants ${ }^{4-8}$. However, to date no study has systematically evaluated the burden of rare (minor allele frequency $<0.01$ ) damaging variants in a "hypothesisfree" approach with functional insights, that could help to expand the genetic architecture of PD, early-onset PD (EOPD) and late-onset PD (LOPD), in order to potentially uncover novel susceptibility genes and pathways.

In the present study, we used two case-control WES cohorts from the International Parkinson's disease Genomics Consortium (IPDGC, www.pdgenetics.org) ${ }^{5}$ and the Parkinson's Progression Markers Initiative (PPMI, www.ppmi-info.org) ${ }^{9}$, and conducted a systematic assessment of the burden of variants across different allele frequencies, functional impact predictions and different ages of onset (AOO) at the whole-exome, gene-set and gene-wise levels in idiopathic PD cases compared to healthy controls. Using a large compendium of functional gene-sets, molecular pathways, tissue and cell-type specific gene expression profiles, and protein-protein interaction 
(PPI) networks, our results revealed a significant burden of damaging ultra-rare variants (URVs) in EOPD cases that are enriched in disease-relevant molecular pathways, and suggest major differences in the genetic and functional architecture compared to LOPD.

\section{RESULTS}

\section{Exome-wide contribution of rare damaging coding variants to Parkinson's disease}

We systematically assessed the contribution of coding variants to PD in two independent casecontrol cohorts of European descent: (i) IPDGC ${ }^{5}$, comprising 1,042 PD cases and 452 controls, and (ii) $\mathrm{PPMI}^{9}$, with $383 \mathrm{PD}$ cases and 144 controls (See complete pipeline in Supplementary Fig. 1). We also stratified cases according to AOO (Table 1). The IPDGC cases had a mean AOO of 43.02 years $(\mathrm{SD}=10.63)$, including $438 \mathrm{EOPD}(\mathrm{AOO}<40$ years of age) and $604 \mathrm{PD}$ cases above 40 years of age $(40+)$. Controls had average age of recruitment of 46.20 years $(\mathrm{SD}=26.07)$. The PPMI cases had a mean AOO of 61.62 years $(\mathrm{SD}=9.72)$, and comprised 9 EOPD and 374 40+ (including 232 LOPD individuals). Controls had an average age of recruitment of 61.11 years $(\mathrm{SD}=10.18)$. Average per sample transitions/transversions $(\mathrm{Ts} / \mathrm{Tv})$, homozygous/heterozygous (Hom/Het) and insertions/deletions (In/Del) ratios were among expected ranges for both cohorts, respectively (IPDGC=3.2, 1.69, 0.87; PPMI=3.19, 1.82, 0.69).

We classified and counted alleles according to frequency, functional and deleteriousness predictions, in different AAOs in cases and controls (average counts on Supplementary Table 1). With regression analyses we observed a highly significant burden of all three classes of ultra-rare variants in the IPDGC EOPD cases compared to controls (URVs= singleton variants in our cohort, having an allele count of $1 / 2988$ and without reported frequency in gnomAD ${ }^{10}$ ): Protein altering variants $(\mathrm{PAV}), \mathrm{P}=3.95 \times 10^{-26}$, beta $=0.158, \mathrm{SE}=0.015$; Nonsynonymous $(\mathrm{NSN}), \mathrm{P}=7.36 \times 10^{-26}$, 
beta $=0.162, \mathrm{SE}=0.015$; and Loss-of-Function $(\mathrm{LoF}), \mathrm{P}=6.1 \times 10^{-4}$, beta=0.295, $\mathrm{SE}=0.086$ (Fig. 1A,

Supplementary Table 2). LoF variants appeared to have a larger contribution compared to NSN variants in EOPD cases. Similar but weaker results were observed for all classes of URVs in all PD cases, except for LoF variants that did not surpass Bonferroni correction (PAV, $\mathrm{P}=9.17 \times 10^{-18}$, beta $=0.102, \mathrm{SE}=0.012 ; \mathrm{NSN}, \mathrm{P}=2.09 \times 10^{-17}$, beta $=0.105, \mathrm{SE}=0.012 ; \mathrm{LOF}, \mathrm{P}=0.004$, beta $=0.209$, $\mathrm{SE}=0.073)$ as well as in $40+\mathrm{PD}$ cases $\left(\mathrm{PAV}, \mathrm{P}=1.92 \times 10^{-6}\right.$, beta $=0.065, \mathrm{SE}=0.014 ; \mathrm{NSN}$, $\mathrm{P}=2.75 \times 10^{-6}$, beta $=0.066, \mathrm{SE}=0.014 ; \mathrm{LOF}, \mathrm{P}=0.098$, beta $\left.=0.133, \mathrm{SE}=0.08\right)$. After removing lower frequency variants for each category, we observed that the top burden results were for more common allele frequencies (i.e. $>$ singletons) in $40+\mathrm{PD}$ cases $(\mathrm{P}=0.003$, beta $=0.01, \mathrm{SE}=0.003$;

Fig. 1B, Supplementary Table 3), although this did not survive multiple testing correction. We next analyzed synonymous and noncoding variants. While we did not observe increased enrichment across the majority of frequencies and effect classes ("Damaging", CADD >12.37; "Benign”, CADD <12.37), we observed significant URV association signals surviving multiple testing correction in EOPD for damaging and benign synonymous variants $\left(\mathrm{P}=9.13 \times 10^{-16}\right.$, beta $=0.38, \mathrm{SE}=0.048 ; \mathrm{P}=1.80 \times 10^{-15}$, beta $=0.22, \mathrm{SE}=0.028$, respectively), and benign noncoding variants $\left(\mathrm{P}=6.37 \times 10^{-8}\right.$, beta $=0.21, \mathrm{SE}=0.038$; Supplementary Fig. 2, Supplementary Table 4). Similar associations were observed for all PD cases and only damaging synonymous variants for 40+ PD. We next assessed if our URV burden results were biased by an artificial excess of rare variants due to sequencing errors or other hidden confounding factors. We therefore corrected the burden tests for URVs by the total number of singleton variants (regardless of their frequency in gnomAD), and observed that the associations remained significant, we even noted increases in the significance and effect size estimates after correction (URVs not corrected, $\mathrm{P}=2.27 \times 10^{-19}$, beta $=0.06, \mathrm{SE}=0.006$; corrected, $\mathrm{P}=1.43 \times 10^{-26}$, beta $\left.=0.08, \mathrm{SE}=0.008\right)$. Using a stricter read depth 
threshold (DP $>20)$, we reevaluated URVs burden across frequencies and functional annotations, and observed that association signals remained highly significant (Supplementary Table 5).

To further explore increased burden of coding variants in PD, we next analyzed the PPMI cohort. Although no results survived Bonferroni correction, we observed the highest burden for PAVs with frequency $<0.001$ in all $\mathrm{PD}$ cases $(\mathrm{P}=0.01$, beta $=0.02, \mathrm{SE}=0.009)$. Interestingly, the strongest effect size was seen for LoFs with frequency $<0.001$ in LOPD $(\mathrm{P}=0.026$, beta $=0.152, \mathrm{SE}=0.07)$ (Supplementary Fig. 3A, Supplementary Table 6). To explore this further, we repeated the analysis after removing URVs and observed that both the effect size and significance increased $(\mathrm{P}=0.014$, beta $=0.20, \mathrm{SE}=0.08$; Supplementary Fig. 3B, Supplementary Table 7). For synonymous and noncoding variants, no frequency category survived multiple testing correction (Supplementary Fig. 4, Supplementary Table 8).

\section{Functional landscape of PD-associated damaging coding variants.}

Enrichment in gene-sets of highly constrained genes

Using the Sequence Kernel Association Test-Optimal (SKAT-O) ${ }^{11}$, we observed the most significant enrichment for NSN URVs within genes with high probability of being intolerant to heterozygous loss-of-function mutations ( $\mathrm{pLI}>0.9, \mathrm{P}=1.25 \times 10^{-20}$ ) followed by genes highly intolerant to 2 loss-of-function mutations (recessive) ( $\left.\mathrm{pRec}>0.9, \mathrm{P}=1.74 \times 10^{-18}\right)$ and lastly in genes highly tolerant to loss-of-function variation ( $\mathrm{pNull}>0.9, \mathrm{P}=1.35 \times 10^{-9}$ ) (Fig. 2A). For PAVs, the most significant enrichment was observed in $\mathrm{pRec}>0.9\left(\mathrm{P}=2.22 \times 10^{-17}\right)$, followed by $\mathrm{pLI}>0.9$ $\left(6.42 \times 10^{-17}\right)$ and then pNull $>0.9\left(\mathrm{P}=1.65 \times 10^{-11}\right)$. LoF variants showed enrichment only in $\mathrm{pRec}$ $>0.9(\mathrm{P}=0.01)$ and $\mathrm{pNu} 1 \mathrm{>}>0.9$ gene-sets $(\mathrm{P}=0.02)$. For IPDGC $40+\mathrm{PD}$ cases, we analyzed the variants with frequency above singletons and observed a significant enrichment of PAVs and 
NSNs variants across all three categories, with the most significant signal in pRec $>0.9$ genes (PAV, $\mathrm{P}=1.55 \times 10^{-5}$; NSN, $\mathrm{P}=3.0 \times 10^{-4}$; Supplementary Fig. 5A). For the PPMI cohort, no significant associations were observed after multiple testing correction (Supplementary Table 9); however, the top signals P-values were observed in LoFs with frequency above rare singleton in pRec $>0.9$ genes analyzed in 40+ PD cases, and in LoFs with frequency $<0.001$ without URVs in pNull $>0.9$ genes analyzed in LOPD cases.

\section{Enrichment in molecular gene-sets and pathways}

Using the Hallmark MsigDB ${ }^{12}$ gene-sets, we observed significant enrichment in NSN URVs within genes up-regulated in response to ultra-violet radiation (UV) (MsigDB name=M5941, $\mathrm{P}=3.58 \times 10^{-6}$; Fig. 2B upper panel), followed by the Mitotic Spindle (M5893, $\mathrm{P}=2.68 \times 10^{-4}$ ). For PAVs, we observed enrichment in genes found in the Apical Junction pathways (M5915, $\left.\mathrm{P}=4.46 \times 10^{-4}\right)$. In the $\mathrm{C} 2$ gene-sets, the top enrichment was seen for PAV URVs within the Immune System pathway (M1045, $\mathrm{P}=1.84 \times 10^{-7}$, Fig. 2B lower panel) and the Reactome Metabolism of Lipids and Lipoproteins (M27451, $\left.\mathrm{P}=2.86 \times 10^{-5}\right)$. For NSN URVs, the NABA Matrisome pathway (M5889, $\left.\mathrm{P}=2.28 \times 10^{-7}\right)$ was observed to be enriched. For less rarer variants in IPDGC 40+ PD cases (frequency $>$ singletons), the top enriched Hallmark pathway was for NSNs within the Bile Acid Metabolism (M5948, $\mathrm{P}=1.63 \times 10^{-8}$ ), and for the $\mathrm{C} 2$ gene-set, the NABA extra cellular membrane Affiliated Proteins pathway (M5880, $\left.\mathrm{P}=6.03 \times 10^{-8}\right)$. Different pathways were highlighted between the Hallmark and C2 gene-sets, with the only overlap found in the C2 NABA Matrisome pathway (M5889, $\mathrm{P}=4.67 \times 10^{-6}$, Supplementary Fig. 5B lower panel). No significant results were observed for variants in 40+ PD (frequency $>$ singletons) and LOPD (frequency $<0.001$ without URVs) in the PPMI dataset (Supplementary Table 10). Previous reports that had performed pathway 
burden analyses have found enrichment of rare damaging NSN variants in both lysosomal storage $\operatorname{disorder}^{7}(\mathrm{LSD})$ genes (frequency $<0.03$ ) and mitochondrial DNA maintenance pathway genes (frequency $<0.01)^{13}$. To validate our hypothesis-free methodology, we performed burden analysis on these two specific pathways. We found that URVs were significantly enriched in the mitochondrial DNA maintenance on EOPD individuals $(\mathrm{P}=0.016)$. We also observed that the LSD genes enrichment was only significant when using more common variants (frequency $<0.05$ ) and that the association was driven by $40+\mathrm{PD}$ cases $\left(\mathrm{P}=1.67 \times 10^{-4}\right.$, Supplementary Table 11).

Enrichment in highly expressed genes across specific tissues from GTEx

We observed significant enrichment signals for genes harboring URVs in EOPD individuals in all GTEx brain tissues ${ }^{14}$, for all three types of variant categories (Fig. 2 C). Interestingly, significant enrichment of PAVs and NSN variants was also detected in several other tissues. For 40+ PD cases, we observed a different landscape for PAVs and NSNs, with no enrichment surpassing multiple testing correction in any brain tissue. For LoF variants the enrichment was significant in other tissues suggesting a distinct contribution compared to the observations in EOPD (Supplementary Fig. 5C). No significant results were observed in the PPMI dataset (Supplementary Table 12).

\section{Protein-protein interaction networks and single-cell enrichment analysis of gene-wise burden}

Gene-wise burden and PPI analysis

Although no genes survived multiple-testing correction, we carried out a protein-protein interaction (PPI) network analysis to explore all nominally significant genes (unadjusted SKAT- 
O P $<0.05$, n=308; Supplementary Table 13) in EOPD and 40+ PD cases. Leveraging both WebgestaltR ${ }^{15}$ and $\mathrm{STRING}^{16}$, we observed a significant network (STRING $\mathrm{P}=0.01$, Supplementary Fig. 6). No significant gene ontology (GO) enrichment was observed, however, the top GO term detected was the Regulation of Microtubule Cytoskeleton Organization (GO:0070507) which was driven by nine URV containing genes: AKAP9, APC, CAMSAP2, CDK5RAP2, CEP120, CKAP2, CYLD, KIF11 and TPR (Supplementary Table 14). After including 25 known and suggested PD genes ${ }^{17}$ (Supplementary Table 15), the network significance dramatically increased (STRING $\mathrm{P}<1 \times 10^{-16}$, Fig. 3A). Within this network, we observed significant GO enrichment within the Negative Regulation of Neuron death pathway (GO:1901215, FDR=4.93×10-6; Supplementary Table 16), containing 7 genes harboring URVs: FGF8, HYOU1, ITSN1, PPP5C, MAP3K5, NAE1, and SIRT1. We next repeated this analysis, but now including 303 genes tagged by the 90 PD GWAS loci ${ }^{1}$, which also yielded a significant network (STRING P $<1 \times 10^{-16}$, Supplementary Fig. 7). Within this network, the most significant specific GO biological process was the Intrinsic apoptotic signaling pathway in response to endoplasmic reticulum (ER) stress (GO:0070059, FDR $=4.2 \times 10^{-3}$, Supplementary Table 17) containing five genes harboring URVs (CREB3, ERN1, HYOU1, MAP3K5, and SIRT1).

\section{Enrichment in specific brain cell types}

We next aimed to identify specific mouse brain cell-types expressing the 308 URV harboring genes using $\mathrm{EWCE}^{18,19}$. We observed significant enrichment on the following three cell types (FDR P <0.05): serotonergic neurons; endothelial mural cells, and vascular leptomeningeal cells

(Fig. 3 B). These results show that URVs could be playing an important role in specific cell-types within the brain. 


\section{Heritability of URVs in PD}

Heritability estimation in EOPD using SingHer ${ }^{20}$, showed that URVs account for $15.9 \%$ of the genetic component, $4.6 \%$ to All PD cases and 2.2\% to 40+ PD. These results support our previous findings regarding the difference of URVs between different AOOs in PD.

\section{DISCUSSION}

Here we have comprehensively studied the burden of rare variants in PD using WES datasets from 1,425 idiopathic PD cases and 596 healthy controls. We have found an increased burden of damaging URVs across affected cases, and observed the highest significance in EOPD individuals, suggesting that some of the missing heritability of EOPD may be accounted for by private variants. URVs have been increasingly gaining attention due to the explosion of NGS-based studies, and have been shown to have a major impact in gene-expression regulation, explaining near $\sim 25 \%$ of the heritability ${ }^{20}$. Similar increased URVs burdens have been seen in schizophrenia ${ }^{21}$, epilepsy ${ }^{22}$ and Alzheimer's disease ${ }^{23}$, being identified as an important source of genetic risk. Here, we estimated that URVs contribute $\sim 15 \%$ towards the heritability of PD which is more than half of the current heritability estimates derived from GWAS identified common variants $(\sim 26 \%)^{1}$. This indicates that URVs may play a major sizeable role in PD etiology thereby warranting further research.

Unique patterns of constrained gene-set enrichment were observed for EOPD and 40+ PD/LOPD. URVs in EOPD predominantly mapped to highly constrained genes ( $\mathrm{pLI}>0.9$ ) suggesting that EOPD may be driven by hetero- and homozygous highly deleterious private variants/URVs. In contrast, 40+ PD and LOPD appear to be driven by more common/infrequent variants (frequency $>$ rare singletons) in genes that are intolerant to homozygous LoF variants mostly (pRec >0.9). 
Their presence within the general population suggests that these variants may be less damaging or have incomplete penetrance, further complicating variant discovery efforts. Our results therefore support previous assertions that different genes/variants and inheritance patterns contribute to EOPD and LOPD $^{24}$. While EOPD-specific molecular signatures have recently been uncovered ${ }^{25}$, more studies are needed to investigate the different etiologies in EOPD and LOPD.

While it is premature to comment on the functional pathways highlighted in the 40+ PD and LOPD, many of the pathways pulled out in the EOPD analysis are of interest. Much research has investigated the proposed genetic link between PD, pigmentation (peripheral melanin and neuromelanin) and melanoma ${ }^{26,27}$, therefore the identified high burden of EOPD URVs in the MsigDB hallmark UV response pathway further highlights the link between PD and melanoma, and additionally implicates a role for pigmentation in disease pathology. The mitotic spindle pathway is associated with microtubule stabilization, a process known to be dysregulated in $\mathrm{PD}^{28}$. Recent evidence has shown that dopamine deficiency (a hallmark of PD) perturbs circadian/mitotic gene networks resulting in increased expression of mitotic spindle pathway genes in the striatum of PD patients ${ }^{29}$. Our observations of increased enrichment of URVs in genes involved in this pathway adds support to its potential role in PD etiology. The apical junction pathway is composed of genes implicated in cell-cell contacts, which are structurally important for different biological functions, such as the intestinal epithelial barrier ${ }^{30}$. Structural alterations of the intestinal epithelial barrier have been found in PD patients ${ }^{31}$, and in Crohn's disease - both of which have LRRK2 as common genetic factor ${ }^{32}$. Thus, our findings of high burden of URVs in the apical junction pathway may provide some evidence for the observed link between the etiology of PD and the gastrointestinal track. URVs enriched in pathways relating to the Immune System and the Metabolism of Lipids and Lipoprotein are interesting targets for functional validation, since they 
are established biological processes affected in PD pathophysiology $y^{33,34}$. The enrichment observed in the Matrisome pathway is of note, since constituent genes are key components of the extra cellular matrix (ECM). Functional and structural damage to the ECM during aging has been observed $^{35}$, impacting important brain structures such as the blood-brain barrier ${ }^{36}$, which is known to be affected in both PD and Alzheimer's disease ${ }^{37}$. The distinct pattern of enrichment of URV harboring genes in brain tissues provide some evidence of their possible functional role, along with the pathways found. Intriguingly, there is again no overlap in the enrichment in EOPD compared to $40+$ PD cases, showing different pathways which reinforce the notion of distinct genetic architectures between AOOs. It is of note that for 40+ PD cases, the enrichment found in nonbrain regions suggests that the roles of the genes/variants involved in LOPD pathogenesis may not be restricted to the brain, which is in agreement with recent evidence indicating that PD risk loci genes do not lie only in specific brain cell types or regions, but are involved in global cellular processes detectable across other tissues/organs ${ }^{38}$.

Although not a single gene survived multiple testing correction, several genes with suggestive enrichment of URVs are interesting candidates. SLC39A1 mutations have been found to disrupt manganese homeostasis and cause childhood-onset parkinsonism-dystonia ${ }^{39}$. SRGAP3 is differentially expressed in dopaminergic $\mathrm{SN}$ neurons ${ }^{40}$, and it is part of the axon guidance pathway already implicated in $\mathrm{PD}^{41,42}$. FGF8 improves dopaminergic cell survival and functional restoration in a rat PD model ${ }^{43}$. HYOU1 plays an important role in hypoxia-induced apoptosis as it accumulates within the endoplasmic reticulum (ER) under hypoxic conditions ${ }^{44}$ with its suppression associated with accelerated cell death ${ }^{45}$. The significant PPI networks identified here (GO:1901215, 0070059), while adding strength to our approach, further highlight the important roles that neuronal death regulation and ER stress play in PD etiology. Much research has linked 
ER stress response to PD pathogenesis (reviewed in Colla, 201946), and pathways related to ER stress such as the unfolded protein response are strongly differentially expressed in prefrontal cortex of PD patients ${ }^{47}$. Several highly interconnected genes within these networks are of interest: SIRT1 (11 interactions) has been shown to be protect against PD in cellular and animal models ${ }^{48}$; MAP3K5 (4 interactions) is inhibited by DJ-1 (PARK7) ${ }^{49}$; and ITSN1 (4 interactions) is involved in endosomal and lysosomal trafficking ${ }^{50}$, which contributes to PD risk through increased $\operatorname{expression}^{51}$.

At the cell-type level, the significant enrichment observed in endothelial-mural cells (vascular smooth muscle cells and pericytes that constitute key structures of the blood-brain barrier ${ }^{52}$ ) is in line with our observations of high burden of URVs in the Matrisome pathway. The observed enrichment in serotoninergic neurons gives novel insights into previous genetic observations of serotonergic dysfunction in PD, where $S N C A$ p.Ala53Thr carriers have reduced brain serotonin transporters $^{53}$ and LRRK2 mutation carriers have increased serotonin transporters in the brain ${ }^{54}$. Enrichment in vascular leptomeningeal cells in conjunction with recent GWAS findings ${ }^{55}$, suggests that both common and URVs within genes important for brain repair mechanisms and neurogenesis ${ }^{56}$ play a role in PD pathology. Together, these data help us expand our understanding of the disease etiology and the role of URVs in PD pathogenesis.

The main limitation of this work relates to insufficient statistical power to detect variant burden and the lack of appropriate age-matched replication cohorts, despite the fact that the IPDGC and PPMI PD cohorts are some of the largest WES datasets available. Not only do we expect that larger genetic cohorts will help replicate our findings, they would also edge this study closer to the prohibitively large sample sizes that are currently needed to achieve the sufficient power. Our observations that both synonymous and noncoding variants appear enriched in PD suggest that our 
findings could be inflated by a global spurious excess of singleton variants within our datasets. However, our strict QC (Ts/Tv ratios within acceptable ranges) and the fact that after correcting the URV burden for the total number of singletons our effect sizes increased and gained significance argue against this. Synonymous and noncoding variants are known to contribute to disease pathology through a wide variety of mechanisms, mostly associated to the regulation of gene expression through disruption of transcription factor binding sites, enhancers and splicing sites $^{57-59}$. It is therefore plausible that these variants are not completely neutral and may contribute to PD etiology, however, more studies are needed to accurately elucidate their role in PD.

Overall, this study shows that URVs have a major contribution to PD, especially in early-onset individuals which have a distinct genetic background from sporadic cases. The identified URVs are significantly enriched in disease relevant gene-sets, pathways and genes that interact with each other as well as with known and suggested PD genes. Our results help to broad the understanding of PD genetic risk load in different age of onset and expand the landscape of biological pathways potentially involved in this devastating disease.

\section{METHODS}

\section{Ethical Statement}

This study was approved by the corresponding local ethical scientific committees. More detailed data usage authorizations are provided elsewhere ${ }^{5,9}$.

\section{Samples used in the study}

International Parkinson's Disease Genomics Consortium (IPDGC) WES cohort. The IPDGC WES data used consisted of a total number of 1,933 self-reported European individuals, composed 
of 1,398 PD cases and 535 neurologically healthy controls (https://pdgenetics.org/resources). A more detailed description of the cohort is reported elsewhere ${ }^{5}$.

Parkinson's Progression Markers Initiative (PPMI) WES study. The PPMI WES dataset includes 645 individuals, composed of 462 PD cases and 183 healthy controls, all of self-reported European descent. Data were obtained from the PPMI database under the appropriate PI membership (www.ppmi-info.org/data) ${ }^{9}$.

\section{Data processing and quality controls}

WES data generation and processing details for both IPDGC and PPMI cohorts have been previously described ${ }^{5,9}$. All analyses were performed using VCF files acquired through authorized requests.

For both cohorts, following multiallelic variant splitting and left normalization of insertion/deletions (indels) with BCFTools ${ }^{60}$, we removed variants with genotype quality (GQ) $<20$, read depth $(\mathrm{DP})<8$, call rate $(\mathrm{CR})<90 \%$, Hardy-Weinberg equilibrium $\mathrm{p}<0.000001$ and monomorphic sites. Then, we removed individuals with (i) $>5 \%$ missing genotype call rates, (ii) cryptic relationships (pi hat $>0.125$ ) and (iii) high rates of genotype heterozygosity $(>5$ standard deviations). We also removed all non-European individuals as determined by principal component analysis (PCA) using SMARTPCA ${ }^{61,62}$ with the 1000 genome project phase 3 used as a reference population $^{63}$. Subjects with established pathogenic variants in known Parkinson's disease genes (LRRK2, SNCA, PINK1, VPS35, PARK2 and DJ-1) were detected and excluded from in both datasets. Following QC, we obtained 1,494 IPDGC samples (1,042 PD cases, 452 controls) and 527 PPMI samples (383 PD cases, 144 controls) with a total of 366,746 and 412,223 variants 
respectively. We performed Transition/Transversion (Ts/Tv), Heterozygous/Homozygous (Het/Hom) and Insertion/Deletion (In/Del) ratios calculations with SnpSift V.40 ${ }^{64}$.

\section{Variant annotation and selection}

Variants were annotated using ANNOVAR ${ }^{65}$, and categorized in two ways. First, using variant frequency data within each cohort and from the gnomAD database ${ }^{10}$, variants were labelled according to their observed alternative allele frequency as being infrequent $(<5 \%)$, rare $(<1 \%)$, very rare $(<0.1 \%)$, singletons (seen in a single carrier in the cohort, but also in gnomAD) and URVs (singletons absent from gnomAD). Second, using variant functional prediction categories, variants were stratified into (a) synonymous, (b) noncoding (c), nonsynonymous (NSN), (d) lossof-function (LoF) (frameshift/non-frameshift In/Dels, stop gains/losses and splicing) and (e) protein altering (PAVs, encompassing NSN and LOF variants). We kept PAVs, NSN and LoF variants that were predicted to be damaging by the CADD algorithm ${ }^{66}$ (score $\geq 12.37$ ). For synonymous and noncoding variants, we used variants with CADD $<12.37$ as "benign" and $>$ 12.37 as "damaging".

\section{Whole-exome, gene-set and gene-wise burden analysis}

All burden analyses were performed on all PD cases and controls, and then stratified for EOPD (AOO $<40$ years old), older than 40 years $(40+\mathrm{PD})$ and LOPD (AOO $>60$ years old), in order to assess the genetic profiles across different AOO groups. All burden tests were performed for each variant frequency/functional category using logistic regression with the $g l m$ R package ${ }^{67}$. Here, we modeled the variant allele counts across the entire exome for each individual with disease status, 
adjusting for sex, population structure (PC1-PC5) and capture metrics (10x percentage of exome coverage).

For the gene-set analysis, we used SKAT-O R package ${ }^{11}$, on the most significant whole-exome burden categories detected, and we grouped variants according to different gene-set categories. First, we used gene constraint metrics from the gnomAD database. We selected all variants within genes with high pLI, $p$ Rec and pNull scores $(\mathrm{pLI}>0.9, \mathrm{n}=3,230 ; \mathrm{pRec}>0.9, \mathrm{n}=4,510 ; \mathrm{pNu}$ ll $>0.9$, $\mathrm{n}=2,096$ ). Second, we used the following gene-set definitions from the Molecular Signatures database v.6.2 (MsigDB) ${ }^{12}$ : (a) The Hallmark gene-sets composed of 50 gene groups that summarize and represent specific well-defined biological states or processes and display coherent expression. These gene sets were generated by a computational methodology based on identifying overlaps between gene sets in other MSigDB collections and retaining genes that display coordinate expression; (b) The C2 gene-set, composed of 4,762 sets curated from various sources such as online pathway databases, the biomedical literature, and knowledge of domain experts. Third, we used gene expression data from 54 tissues from the GTEx v.8 database ${ }^{14}$ (gene median transcripts per million per tissue). We selected genes where expression in a given tissue was five times higher than the median expression across all tissues, as previously used ${ }^{68}$. We obtained SKAT-O association P-values for each gene-set after correction for multiple testing using a familywise error rate $($ FWER $<0.05)$, calculated based on 10,000 permutations.

For the gene-wise analysis, we used SKAT-O with the same covariates as the gene-set approach and correcting for multiple testing using FWER $<0.05$. Genes with at least two carriers and an uncorrected P-value of $<0.05$ were kept for further PPI network and single-cell RNASeq enrichment analyses. 


\section{Protein-protein interaction network analysis and single-cell enrichment of gene-wise burden}

\section{candidates}

To study the interplay between proteins coded by genes prioritized by the gene-wise burden analysis, we utilized the R package WebgestaltR ${ }^{15}$ to build PPI networks via network topology analysis and random walk algorithm. Using the Network Retrieval and Prioritization mode, we submitted the list of genes in different combinations: first, the gene-wise burden candidates list alone (Supplementary Table 13), then adding 26 known and suggested EOPD genes (Supplementary Table 15) and finally including 303 genes of interest tagged by the 90 risk loci coming from the recent PD GWAS meta-analysis ${ }^{1}$. GO analysis enrichment were obtained from the Biogrid PPI Networks database ${ }^{69}$. FDR adjustment was used to correct for multiple testing. In order to obtain PPI enrichment statistics, we submitted the same combination of genes to STRING $^{16}$, and retrieved high confidence networks. For visualization and feature annotation purposes we loaded the resulting networks to Cytoscape v.3.7.2 $2^{70}$.

To observe if genes coming from the gene-wise burden analyses play a role in specific brain cell types, we used the Expression Weighted Cell Type Enrichment analysis (EWCE) ${ }^{18}$ with singlecell and single-nuclei RNASeq expression datasets from 24 brain cell types from mice ${ }^{55}$. Briefly, the EWCE pipeline identifies mouse orthologs (if available), and then tests whether genes have higher expression levels in a given cell type than can reasonably be expected by chance. The enrichment tests are corrected using the Benjamini-Hochberg FDR method.

\section{Heritability estimation of ultra-rare variants}

We used the Singleton Heritability inference with REML (SingHer) R package ${ }^{20}$ to calculate the heritability of URVs in EOPD, all PD cases and 40+ in the IPDGC cohort. 


\section{ACKNOWLEDGEMENTS}

We would like to thank the all the patients that provided their genetic information. For IPGCG acknowledgements please visit https://pdgenetics.org/partners.

\section{AUTHOR CONTRIBUTIONS}

B.I.B. and S.J.L. conceived and designed the experiments. B.I.B and S.J.L. performed experiments or analyzed the data. D.K. and IPDGC members helped in drafting the manuscript. All authors reviewed the manuscript for scientific accuracy.

\section{DATA AVAILABILITY}

IPDGC and PPMI whole-exome sequencing data are publicly available upon application through their respective project websites (https://pdgenetics.org/resources, http://www.ppmiinfo.org/access-data-specimens/download-data/)

\section{FUNDING}

This work was supported by the Simpson Querrey Center for Neurogenetics (to D.K.)

\section{COMPETING INTERESTS}

D.K. is the Founder and Scientific Advisory Board Chair of Lysosomal Therapeutics Inc. D.K. serves on the scientific advisory boards of The Silverstein Foundation, Intellia Therapeutics, and Prevail Therapeutics and is a Venture Partner at OrbiMed. B.I.B and S.J.L. declare that they have no competing interests. 


\section{REFERENCES}

1 Nalls, M. A. et al. Identification of novel risk loci, causal insights, and heritable risk for Parkinson's disease: a meta-analysis of genome-wide association studies. Lancet Neurol 18, 1091-1102, doi:10.1016/S1474-4422(19)30320-5 (2019).

2 Klein, C. \& Westenberger, A. Genetics of Parkinson's disease. Cold Spring Harb Perspect Med 2, a008888, doi:10.1101/cshperspect.a008888 (2012).

3 Olgiati, S., Quadri, M. \& Bonifati, V. Genetics of movement disorders in the next-generation sequencing era. Mov Disord 31, 458-470, doi:10.1002/mds.26521 (2016).

4 Farlow, J. L. et al. Whole-Exome Sequencing in Familial Parkinson Disease. JAMA Neurol 73, 68-75, doi:10.1001/jamaneurol.2015.3266 (2016).

5 Jansen, I. E. et al. Establishing the role of rare coding variants in known Parkinson's disease risk loci. Neurobiol Aging 59, 220 e211-220 e218, doi:10.1016/j.neurobiolaging.2017.07.009 (2017).

6 Jansen, I. E. et al. Discovery and functional prioritization of Parkinson's disease candidate genes from large-scale whole exome sequencing. Genome Biol 18, 22, doi:10.1186/s13059-017-11479 (2017).

7 Robak, L. A. et al. Excessive burden of lysosomal storage disorder gene variants in Parkinson's disease. Brain 140, 3191-3203, doi:10.1093/brain/awx285 (2017).

8 Yemni, E. A. et al. Integrated Analysis of Whole Exome Sequencing and Copy Number Evaluation in Parkinson's Disease. Sci Rep 9, 3344, doi:10.1038/s41598-019-40102-x (2019).

9 Parkinson Progression Marker, I. The Parkinson Progression Marker Initiative (PPMI). Prog Neurobiol 95, 629-635, doi:10.1016/j.pneurobio.2011.09.005 (2011).

10 Karczewski, K. J. et al. Variation across 141,456 human exomes and genomes reveals the spectrum of loss-of-function intolerance across human protein-coding genes. bioRxiv, 531210, doi:10.1101/531210 (2019).

$11 \mathrm{Lee}, \mathrm{S}$. et al. Optimal unified approach for rare-variant association testing with application to small-sample case-control whole-exome sequencing studies. Am J Hum Genet 91, 224-237, doi:10.1016/j.ajhg.2012.06.007 (2012).

12 Subramanian, A. et al. Gene set enrichment analysis: a knowledge-based approach for interpreting genome-wide expression profiles. Proc Natl Acad Sci U S A 102, 15545-15550, doi:10.1073/pnas.0506580102 (2005).

13 Gaare, J. J. et al. Rare genetic variation in mitochondrial pathways influences the risk for Parkinson's disease. Mov Disord 33, 1591-1600, doi:10.1002/mds.64 (2018).

14 Aguet, F. et al. The GTEx Consortium atlas of genetic regulatory effects across human tissues. bioRxiv, 787903, doi:10.1101/787903 (2019).

15 Liao, Y., Wang, J., Jaehnig, E. J., Shi, Z. \& Zhang, B. WebGestalt 2019: gene set analysis toolkit with revamped UIs and APIs. Nucleic Acids Res 47, W199-W205, doi:10.1093/nar/gkz401 (2019).

16 Szklarczyk, D. et al. STRING v11: protein-protein association networks with increased coverage, supporting functional discovery in genome-wide experimental datasets. Nucleic Acids Res 47, D607-D613, doi:10.1093/nar/gky1131 (2019).

17 Blauwendraat, C., Nalls, M. A. \& Singleton, A. B. The genetic architecture of Parkinson's disease. Lancet Neurol 19, 170-178, doi:10.1016/S1474-4422(19)30287-X (2020). 
18 Skene, N. G. \& Grant, S. G. Identification of Vulnerable Cell Types in Major Brain Disorders Using Single Cell Transcriptomes and Expression Weighted Cell Type Enrichment. Front Neurosci 10, 16, doi:10.3389/fnins.2016.00016 (2016).

19 Zeisel, A. et al. Brain structure. Cell types in the mouse cortex and hippocampus revealed by single-cell RNA-seq. Science 347, 1138-1142, doi:10.1126/science.aaa1934 (2015).

20 Hernandez, R. D. et al. Ultrarare variants drive substantial cis heritability of human gene expression. Nat Genet 51, 1349-1355, doi:10.1038/s41588-019-0487-7 (2019).

21 Genovese, G. et al. Increased burden of ultra-rare protein-altering variants among 4,877 individuals with schizophrenia. Nat Neurosci 19, 1433-1441, doi:10.1038/nn.4402 (2016).

22 Epi25 Collaborative. Electronic address, s. b. u. e. a. \& Epi, C. Ultra-Rare Genetic Variation in the Epilepsies: A Whole-Exome Sequencing Study of 17,606 Individuals. Am J Hum Genet 105, 267-282, doi:10.1016/j.ajhg.2019.05.020 (2019).

23 Raghavan, N. S. et al. Whole-exome sequencing in 20,197 persons for rare variants in Alzheimer's disease. Ann Clin Transl Neurol 5, 832-842, doi:10.1002/acn3.582 (2018).

24 Marder, K. et al. Familial aggregation of early- and late-onset Parkinson's disease. Ann Neurol 54, 507-513, doi:10.1002/ana.10711 (2003).

25 Laperle, A. H. et al. iPSC modeling of young-onset Parkinson's disease reveals a molecular signature of disease and novel therapeutic candidates. Nat Med 26, 289-299, doi:10.1038/s41591-019-0739-1 (2020).

26 Lubbe, S. J. et al. Rare variants analysis of cutaneous malignant melanoma genes in Parkinson's disease. Neurobiol Aging 48, 222 e221-222 e227, doi:10.1016/j.neurobiolaging.2016.07.013 (2016).

27 Dube, U. et al. Overlapping genetic architecture between Parkinson disease and melanoma. Acta Neuropathol 139, 347-364, doi:10.1007/s00401-019-02110-z (2020).

28 Cartelli, D. \& Cappelletti, G. Microtubule Destabilization Paves the Way to Parkinson's Disease. Mol Neurobiol 54, 6762-6774, doi:10.1007/s12035-016-0188-5 (2017).

29 Jiang, P. et al. Parkinson's Disease is Associated with Dysregulations of a DopamineModulated Gene Network Relevant to Sleep and Affective Neurobehaviors in the Striatum. Sci Rep 9, 4808, doi:10.1038/s41598-019-41248-4 (2019).

30 Vogelmann, R., Amieva, M. R., Falkow, S. \& Nelson, W. J. Breaking into the epithelial apicaljunctional complex--news from pathogen hackers. Curr Opin Cell Biol 16, 86-93, doi:10.1016/j.ceb.2003.12.002 (2004).

31 Clairembault, T. et al. Structural alterations of the intestinal epithelial barrier in Parkinson's disease. Acta Neuropathol Commun 3, 12, doi:10.1186/s40478-015-0196-0 (2015).

32 Hui, K. Y. et al. Functional variants in the LRRK2 gene confer shared effects on risk for Crohn's disease and Parkinson's disease. Sci Transl Med 10, doi:10.1126/scitranslmed.aai7795 (2018).

33 Tansey, M. G. \& Romero-Ramos, M. Immune system responses in Parkinson's disease: Early and dynamic. Eur J Neurosci 49, 364-383, doi:10.1111/ejn.14290 (2019).

34 Xicoy, H., Wieringa, B. \& Martens, G. J. M. The Role of Lipids in Parkinson's Disease. Cells 8, doi:10.3390/cells8010027 (2019).

35 Ewald, C. Y. The Matrisome during Aging and Longevity: A Systems-Level Approach toward Defining Matreotypes Promoting Healthy Aging. Gerontology, 1-9, doi:10.1159/000504295 (2019).

36 Joutel, A., Haddad, I., Ratelade, J. \& Nelson, M. T. Perturbations of the cerebrovascular matrisome: A convergent mechanism in small vessel disease of the brain? J Cereb Blood Flow Metab 36, 143-157, doi:10.1038/jcbfm.2015.62 (2016). 
37 Desai, B. S., Monahan, A. J., Carvey, P. M. \& Hendey, B. Blood-brain barrier pathology in Alzheimer's and Parkinson's disease: implications for drug therapy. Cell Transplant 16, 285299, doi:10.3727/000000007783464731 (2007).

38 Reynolds, R. H. et al. Moving beyond neurons: the role of cell type-specific gene regulation in Parkinson's disease heritability. NPJ Parkinsons Dis 5, 6, doi:10.1038/s41531-019-0076-6 (2019).

39 Tuschl, K. et al. Mutations in SLC39A14 disrupt manganese homeostasis and cause childhoodonset parkinsonism-dystonia. Nat Commun 7, 11601, doi:10.1038/ncomms11601 (2016).

40 Elstner, M. et al. Single-cell expression profiling of dopaminergic neurons combined with association analysis identifies pyridoxal kinase as Parkinson's disease gene. Ann Neurol 66, 792-798, doi:10.1002/ana.21780 (2009).

41 Lesnick, T. G. et al. A genomic pathway approach to a complex disease: axon guidance and Parkinson disease. PLoS Genet 3, e98, doi:10.1371/journal.pgen.0030098 (2007).

42 Maraganore, D. M. et al. High-resolution whole-genome association study of Parkinson disease. Am J Hum Genet 77, 685-693, doi:10.1086/496902 (2005).

43 Jensen, P., Pedersen, E. G., Zimmer, J., Widmer, H. R. \& Meyer, M. Functional effect of FGF2and FGF8-expanded ventral mesencephalic precursor cells in a rat model of Parkinson's disease. Brain Res 1218, 13-20, doi:10.1016/j.brainres.2008.04.039 (2008).

44 Ozawa, K. et al. 150-kDa oxygen-regulated protein (ORP150) suppresses hypoxia-induced apoptotic cell death. J Biol Chem 274, 6397-6404, doi:10.1074/jbc.274.10.6397 (1999).

45 Zhao, L., Rosales, C., Seburn, K., Ron, D. \& Ackerman, S. L. Alteration of the unfolded protein response modifies neurodegeneration in a mouse model of Marinesco-Sjogren syndrome. Hum Mol Genet 19, 25-35, doi:10.1093/hmg/ddp464 (2010).

46 Colla, E. Linking the Endoplasmic Reticulum to Parkinson's Disease and AlphaSynucleinopathy. Front Neurosci 13, 560, doi:10.3389/fnins.2019.00560 (2019).

47 Nido, G. S. et al. Common gene expression signatures in Parkinson's disease are driven by changes in cell composition. Acta Neuropathol Commun 8, 55, doi:10.1186/s40478-020-009327 (2020).

48 Herskovits, A. Z. \& Guarente, L. SIRT1 in neurodevelopment and brain senescence. Neuron 81, 471-483, doi:10.1016/j.neuron.2014.01.028 (2014).

$49 \mathrm{Oh}, \mathrm{S}$. E. et al. The Parkinson's disease gene product DJ-1 modulates miR-221 to promote neuronal survival against oxidative stress. Redox Biol 19, 62-73, doi:10.1016/j.redox.2018.07.021 (2018).

50 N, M. et al. The Down Syndrome-Associated Protein, Regulator of Calcineurin-1, is Altered in Alzheimer's Disease and Dementia with Lewy Bodies. J Alzheimers Dis Parkinsonism 9, doi:10.4172/2161-0460.1000462 (2019).

51 Bandres-Ciga, S. et al. The endocytic membrane trafficking pathway plays a major role in the risk of Parkinson's disease. Mov Disord 34, 460-468, doi:10.1002/mds.27614 (2019).

52 Armulik, A. et al. Pericytes regulate the blood-brain barrier. Nature 468, 557-561, doi:10.1038/nature09522 (2010).

53 Wilson, H. et al. Serotonergic pathology and disease burden in the premotor and motor phase of A53T alpha-synuclein parkinsonism: a cross-sectional study. Lancet Neurol 18, 748-759, doi:10.1016/S1474-4422(19)30140-1 (2019).

54 Wile, D. J. et al. Serotonin and dopamine transporter PET changes in the premotor phase of LRRK2 parkinsonism: cross-sectional studies. Lancet Neurol 16, 351-359, doi:10.1016/S14744422(17)30056-X (2017). 
55 Bryois, J. et al. Genetic Identification of Cell Types Underlying Brain Complex Traits Yields Novel Insights Into the Etiology of Parkinson's Disease. bioRxiv, 528463, doi:10.1101/528463 (2019).

56 Rose, S. E. et al. Leptomeninges-Derived Induced Pluripotent Stem Cells and Directly Converted Neurons From Autopsy Cases With Varying Neuropathologic Backgrounds. J Neuropathol Exp Neurol 77, 353-360, doi:10.1093/jnen/nly013 (2018).

57 Zeng, Z. \& Bromberg, Y. Predicting Functional Effects of Synonymous Variants: A Systematic Review and Perspectives. Front Genet 10, 914, doi:10.3389/fgene.2019.00914 (2019).

58 Zhang, F. \& Lupski, J. R. Non-coding genetic variants in human disease. Hum Mol Genet 24, R102-110, doi:10.1093/hmg/ddv259 (2015).

59 Sauna, Z. E. \& Kimchi-Sarfaty, C. Understanding the contribution of synonymous mutations to human disease. Nat Rev Genet 12, 683-691, doi:10.1038/nrg3051 (2011).

$60 \mathrm{Li}, \mathrm{H}$. et al. The Sequence Alignment/Map format and SAMtools. Bioinformatics 25, 20782079, doi:10.1093/bioinformatics/btp352 (2009).

61 Patterson, N., Price, A. L. \& Reich, D. Population structure and eigenanalysis. PLoS Genet 2, e190, doi:10.1371/journal.pgen.0020190 (2006).

62 Price, A. L. et al. Principal components analysis corrects for stratification in genome-wide association studies. Nat Genet 38, 904-909, doi:10.1038/ng1847 (2006).

63 Genomes Project, C. et al. A global reference for human genetic variation. Nature 526, 68-74, doi:10.1038/nature15393 (2015).

64 Cingolani, P. et al. A program for annotating and predicting the effects of single nucleotide polymorphisms, SnpEff: SNPs in the genome of Drosophila melanogaster strain w1118; iso-2; iso-3. Fly (Austin) 6, 80-92, doi:10.4161/fly.19695 (2012).

65 Wang, K., Li, M. \& Hakonarson, H. ANNOVAR: functional annotation of genetic variants from high-throughput sequencing data. Nucleic Acids Res 38, e164, doi:10.1093/nar/gkq603 (2010).

66 Rentzsch, P., Witten, D., Cooper, G. M., Shendure, J. \& Kircher, M. CADD: predicting the deleteriousness of variants throughout the human genome. Nucleic Acids Res 47, D886-D894, doi:10.1093/nar/gky1016 (2019).

67 Team, R. C. R: A Language and Environment for Statistical Computing, 2017).

$68 \mathrm{Zhou}$, J. et al. Whole-genome deep-learning analysis identifies contribution of noncoding mutations to autism risk. Nat Genet 51, 973-980, doi:10.1038/s41588-019-0420-0 (2019).

69 Oughtred, R. et al. The BioGRID interaction database: 2019 update. Nucleic Acids Res 47, D529-D541, doi:10.1093/nar/gky1079 (2019).

70 Shannon, P. et al. Cytoscape: a software environment for integrated models of biomolecular interaction networks. Genome Res 13, 2498-2504, doi:10.1101/gr.1239303 (2003). 


\section{Figure Legends}

Figure 1. Whole-exome burden of rare coding damaging variants in the IPDGC cohort. Burden analysis of different variant frequencies and functional categories for all PD cases, cases over under 40 years old (EOPD) and over 40 years old (40+). Logistic regression - $\log _{10}(\mathrm{P}$-values) and beta coefficients are represented for: A) Burden from variants with 5\% or less frequency to ultra-rare variants (URVs). B) Burden towards more common variants removing lower frequency variants from each variant category. AAF: Alternative allele frequency; PAV: Protein-altering variants; NSN: Nonsynonymous variants; LoF: Loss-of-function variants.

Figure 2. Gene-set burden analysis in the IPDGC cohort for EOPD cases. Gene-set analysis performed with SKAT-O using PAV, NSN and LoF URVs using gene lists from: A) gnomAD gene constraint metrics pLI, pRec and pNull (>0.9); B) MsigDB Hallmark pathways (upper panel) and C2 curated pathways (lower panel); C) High expression genes from 54 tissues in the GTEx database V8. A family-wise error rate $\mathrm{P}<0.05$ was used as a threshold for statistically significant results.

Figure 3. Protein-protein Interaction Network and Single-Cell enrichment analysis of genewise burden results in EOPD. A) Network built using 308 genes with SKAT-O P $<0.05$ from EOPD URVs and 26 known and suggested PD causal genes with WebGestalt Network Topologybased Analysis (NTA). Statistics from STRING using the same list of genes are included. Shades of blue indicate SKATO P-values from less (light-blue) to more significant (dark-blue) burden association. Node size indicate the number of variants observed in each gene and the green 
bioRxiv preprint doi: https://doi.org/10.1101/2020.06.06.137299; this version posted June 6, 2020. The copyright holder for this preprint (which

was not certified by peer review) is the author/funder, who has granted bioRxiv a license to display the preprint in perpetuity. It is made available under aCC-BY-NC-ND 4.0 International license.

perimeter around the nodes show genes members of significant gene-ontology enrichment results.

B) Single-cell and single-nuclei RNASeq enrichment analysis of EOPD gene-wise burden list leveraged using expression datasets from 24 mice brain tissues. Red bars represent significant results surpassing a FDR cutoff of $<0.05$. 
bioRxiv preprint doi: https://doi.org/10.1101/2020.06.06.137299; this version posted June 6, 2020. The copyright holder for this preprint (which was not certified by peer review) is the author/funder, who has granted bioRxiv a license to display the preprint in perpetuity. It is made available under aCC-BY-NC-ND 4.0 International license.

Table 1. Whole-exome cohorts analyzed in the present study

\begin{tabular}{|c|c|c|c|c|c|c|}
\hline $\begin{array}{c}\text { Whole-exome } \\
\text { cohort }\end{array}$ & $\begin{array}{l}\text { Median age of } \\
\text { PD onset }\end{array}$ & $\begin{array}{c}\text { Median age of } \\
\text { recruitment in } \\
\text { controls } \\
\end{array}$ & Burden groups & Sample size & Cases & Controls \\
\hline \multirow{3}{*}{ IPDGC } & \multirow{3}{*}{$43.02( \pm 10.63)$} & \multirow{3}{*}{$46.20( \pm 26.07)$} & All & 1,494 & 1,042 & 452 \\
\hline & & & Cases $<40$ y/o (EOPD) & 890 & 438 & 452 \\
\hline & & & Cases $>40 \mathrm{y} / \mathrm{o}$ & 1,056 & 604 & 452 \\
\hline \multirow{3}{*}{ PPMI } & \multirow{3}{*}{$61.62( \pm 9.72)$} & \multirow{3}{*}{$61.11( \pm 10.18)$} & All & 527 & 383 & 144 \\
\hline & & & Cases $>40 \mathrm{y} / \mathrm{o}$ & 518 & 374 & 144 \\
\hline & & & Cases $>60$ y/o (LOPD) & 376 & 232 & 144 \\
\hline
\end{tabular}


bioRxiv preprint doi: https://doi.org/10.1101/2020.06.06.137299; this version posted June 6, 2020. The copyright holder for this preprint (which was not certified by peer review) is the author/funder, who has granted bioRxiv a license to display the preprint in perpetuity. It is made available under aCC-BY-NC-ND 4.0 International license.

\section{Figure 1.}

A

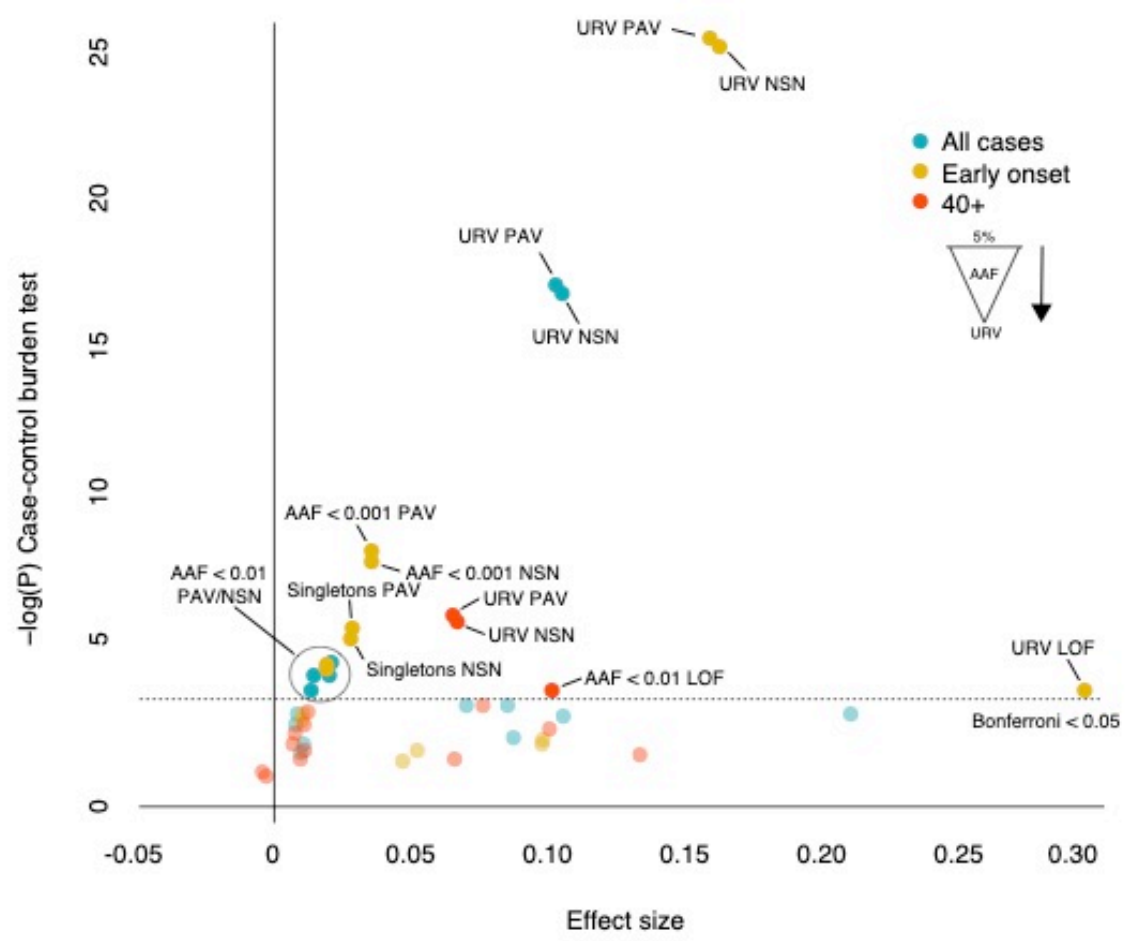

B

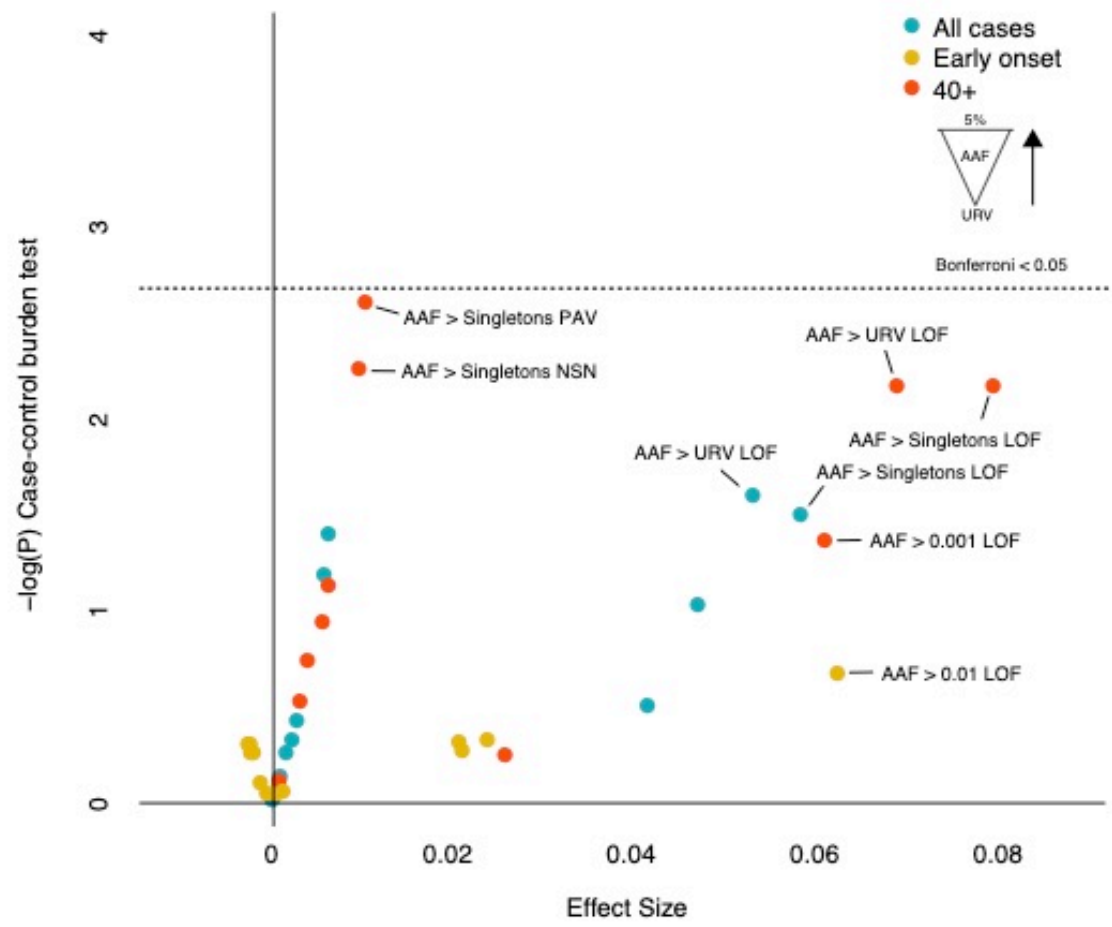


bioRxiv preprint doi: https://doi.org/10.1101/2020.06.06.137299; this version posted June 6, 2020. The copyright holder for this preprint (which was not certified by peer review) is the author/funder, who has granted bioRxiv a license to display the preprint in perpetuity. It is made available under aCC-BY-NC-ND 4.0 International license.

\section{Figure 2.}

A

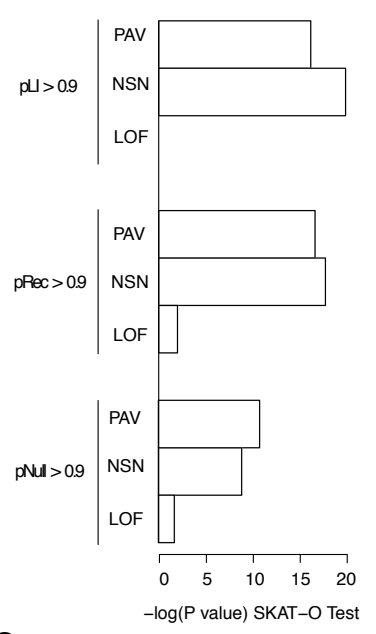

B
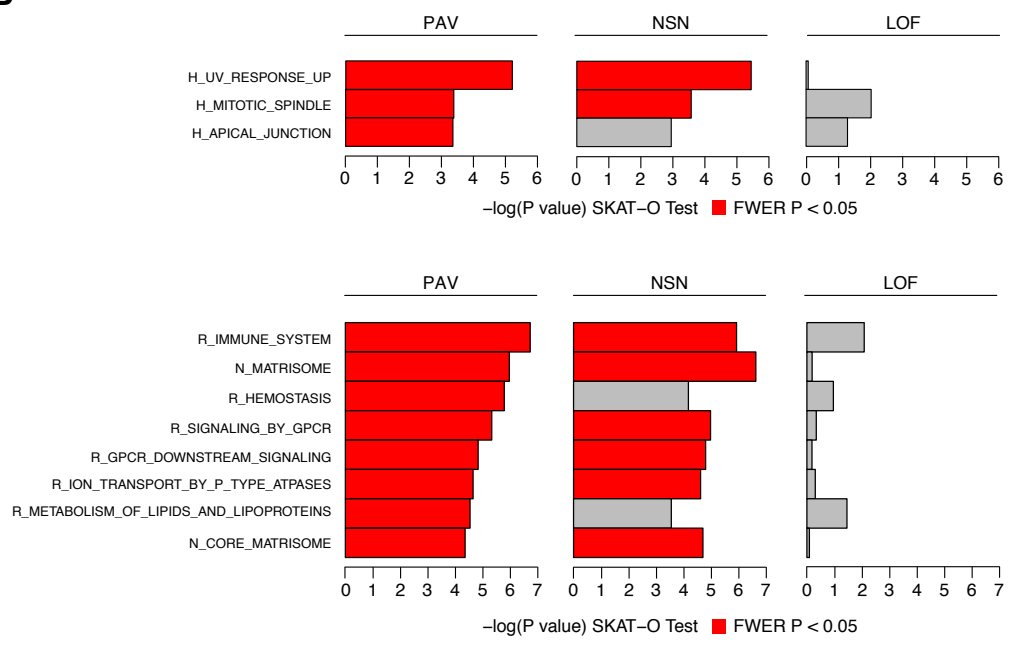

C

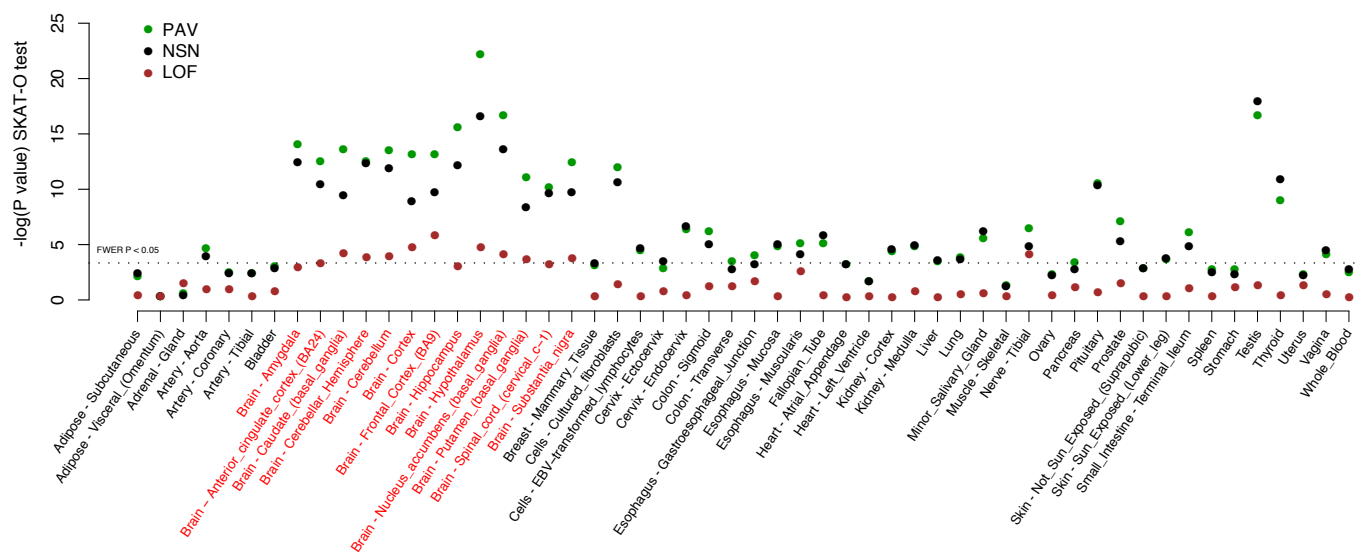


bioRxiv preprint doi: https://doi.org/10.1101/2020.06.06.137299; this version posted June 6, 2020. The copyright holder for this preprint (which was not certified by peer review) is the author/funder, who has granted bioRxiv a license to display the preprint in perpetuity. It is made available under aCC-BY-NC-ND 4.0 International license.

\section{Figure 3.}

A

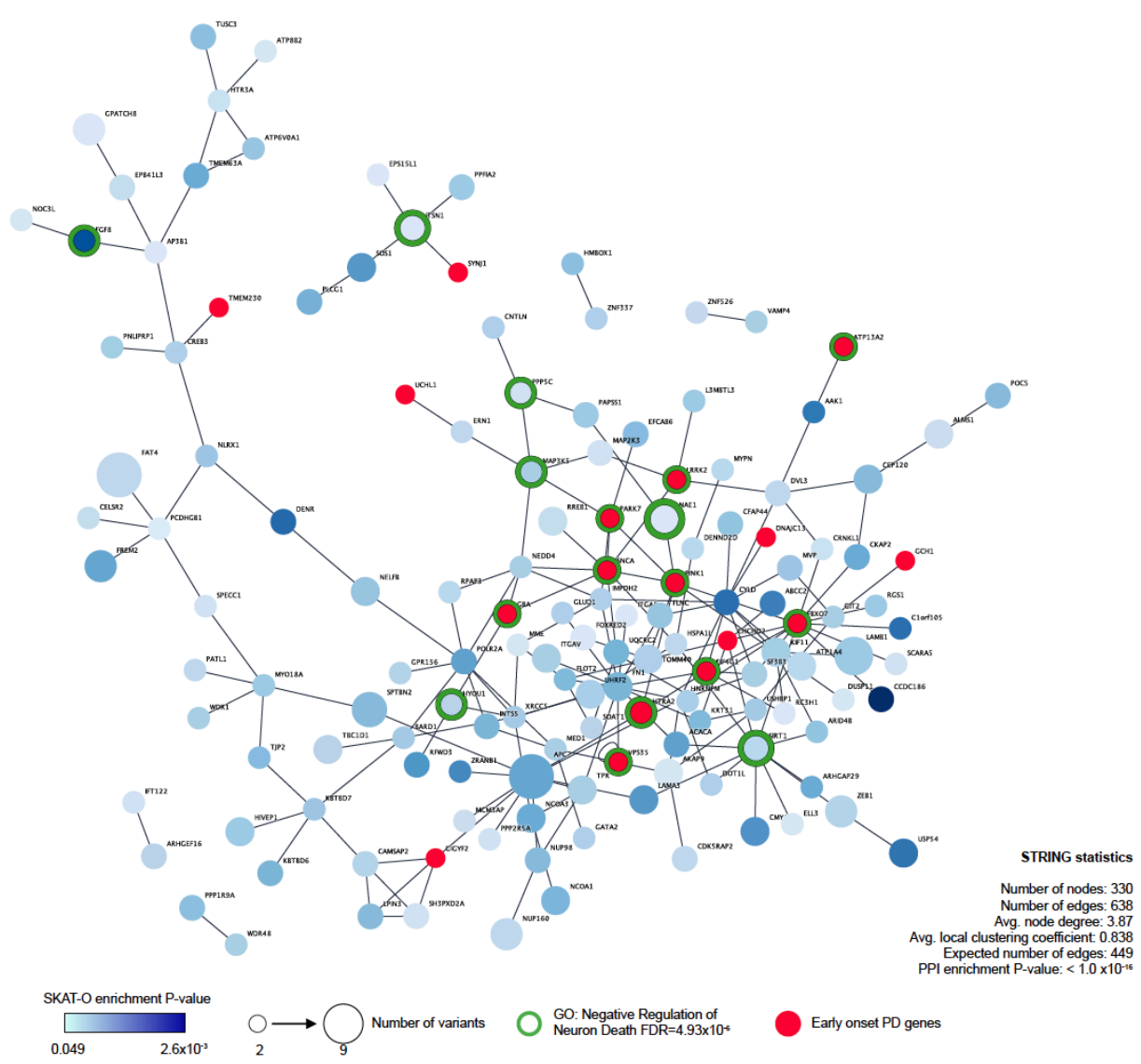

$\mathbf{B}$

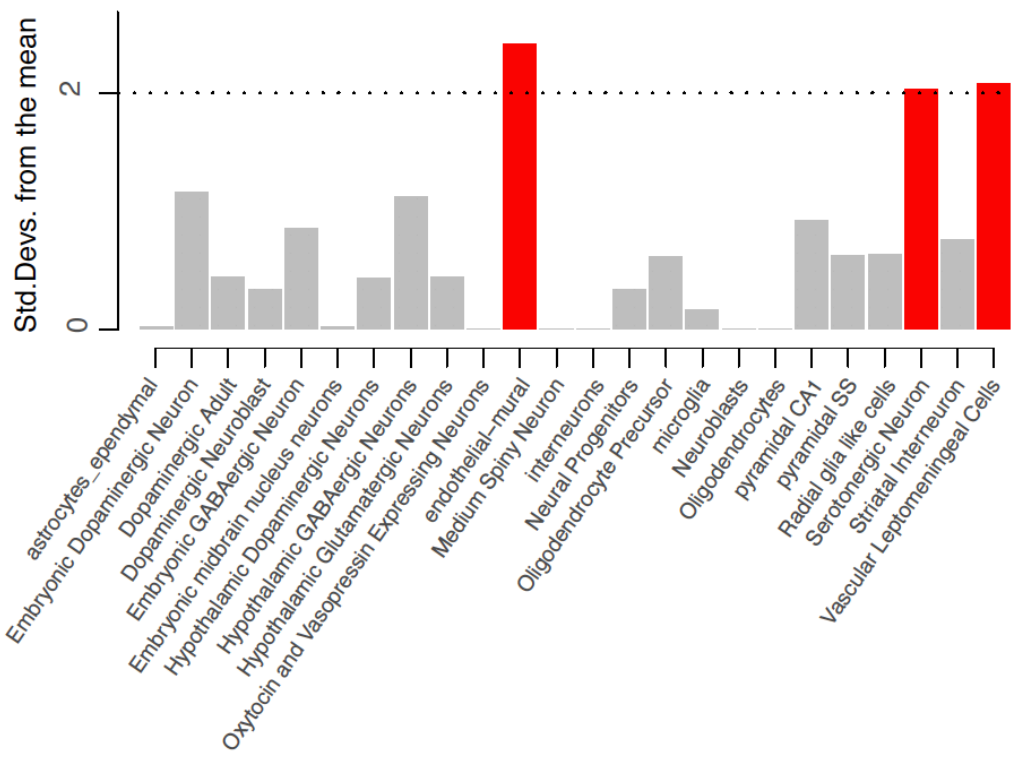

